\title{
Effect of Co and Mn Doping on the Martensitic Transformations and Magnetic Properties of Fe-Pd Ferromagnetic Shape Memory Alloys
}

\author{
Vicente Sánchez-Alarcos ${ }^{1,2,3}$, Jose Ignacio Pérez-Landazábal ${ }^{1, b}$, \\ Vicente Recarte ${ }^{1, c}$ \\ ${ }^{1}$ Departamento de Física, Universidad Pública de Navarra, Campus de Arrosadía 31006 \\ Pamplona, Spain \\ ${ }^{2}$ Instituto de Ciencia de Materiales de Aragón (CSIC-Universidad de Zaragoza), 50009 Zaragoza, \\ Spain \\ ${ }^{3}$ Institut Laue Langevin (ILL), 6 Rue Jules Horowitz, Grenoble, France \\ avicente.sanchez@unavarra.es, bipzlanda@unavarra.es, 'recarte@unavarra.es
}

Keywords: Fe-Pd, Ferromagnetic shape memory alloys, martensitic transformation, Fe-Pd-Mn, Fe-Pd-Co.

\begin{abstract}
The effect of the partial substitution of Fe by Co and Mn on the structural and magnetic properties of Fe-Pd alloys has been investigated by means of calorimetric, magnetic and neutron diffraction measurements. The addition of $\mathrm{Mn}$ increases both the reversible and irreversible MT temperatures whereas the addition of Co causes the opposite effect, which points out that that the compositional dependence of the transformation temperatures of the Fe-Pd alloys is not related to the $\mathrm{Pd}$ amount but to the $e / a$ concentration. Furthermore, it is shown that the appearance of the BCT phase is directly related to the tetragonality of the FCT phase. On the other hand, the amount of FCT martensite that irreversibly transforms into the undesirable BCT phase is considerably reduced by both the $\mathrm{Co}$ and $\mathrm{Mn}$ doping. The substitution of $\mathrm{Fe}$ by $\mathrm{Co}$ seems to be especially interesting for magnetic field-induce strains applications since both the magnetic anisotropy and the saturation magnetization of the Fe-Pd FCT martensite are considerably increased with the Co addition.
\end{abstract}

\section{Introduction}

Ferromagnetic shape memory alloys (FSMA) are being widely studied and discussed along the last two decades because of the unique properties they show as a result of the combination of a thermoelastic Martensitic Transformation (MT) and a magnetic transition. One of the most interesting properties of these functional materials is the extraordinary high magnetoplasticity they show as a consequence of the magnetic field induced re-orientation of the crystallographic domains (so called martensitic variants) appearing as a result of the thermoelastic MT [1]. The possibility of achieving giant magnetic field induced strains (MFIS) have made FSMA to be especially attractive for practical applications such as magnetic actuation, towards most of the works have been traditionally focused (although several and interesting properties such as the magnetocaloric effect and the magnetoresistivity are being recently investigated in FSMA because of its potential applications [2, 3]). Among FSMA, the highest values of MFIS (as large as 10\% [4]) has been obtained in Ni-Mn-Ga alloys close to the stoichiometric Heusler composition $\mathrm{Ni}_{2} \mathrm{MnGa}$, by far the most widely studied FSMA system. However, Ni-Mn-Ga alloys show such a poor mechanical response that the implementation of Ni-Mn-Ga MFIS-based practical devices is almost unfeasible. Therefore, the different alternatives to Ni-Mn-Ga must be considered. In this sense, the research for FSMA systems showing high MFIS and good mechanical properties is still an active topic.

Fe-Pd alloys with compositions close to $\mathrm{Fe}_{70} \mathrm{Pd}_{30}$ show the best mechanical properties among the FSMA as well as MFIS as high as 3.1\%, [5-6], so they seem to be highly suitable for the development of magnetic actuators. Furthermore, the Curie temperature, $\mathrm{T}_{\mathrm{C}}$, of these alloys lies around $600 \mathrm{~K}$ [7] (much above $\mathrm{T}_{\mathrm{C}}$ of the Ni-Mn-Ga alloys, typically around $370 \mathrm{~K}$ ), which can be 
interesting for industrial applications. On cooling, the Fe-Pd alloys with Pd content ranging from $29 \%$ to $32 \%$ undergo a thermoelastic MT from a face-centered cubic austenitic phase (FCC) to a face-centered tetragonal martensite (FCT), at a temperature strongly dependent on the Pd content [8, 9] (a reverse FCT $\rightarrow$ FCC transformation occurs on heating due to the thermoelastic character of the $\mathrm{MT}$ ). Nevertheless, the $\mathrm{FCC} \rightarrow \mathrm{FCT}$ transformation temperatures lie typically below room temperature, which means a serious drawback for most practical application. Furthermore, on further cooling a non-thermoelastic transformation takes place between the FCT martensitic structure and a body-centered tetragonal one (BCT). The FCT $\rightarrow \mathrm{BCT}$ transformation is nonthermoelastic and, therefore, irreversible, in such a way that the appearing BCT phase will remain (thus degrading the shape memory properties) unless the alloy is annealed at high temperature and subsequently quenched. Since the FCT $\rightarrow$ BCT transformation temperature show a similar dependence on the Pd concentration than the FCC $\rightarrow$ FCT one (both of them abruptly decreases with the increasing Pd content), any attempt to decrease the temperature of the undesirable irreversible transformation implies a decrease of the thermoelastic MT temperature. The addition of a doping element could be an effective way of improving the temperature stability range of the thermoelastic martensite, as it occurs in the Ni-Mn-Ga system [10], but, surprisingly, very few works devoted to this subject can be found in the literature. In fact, only the addition of $\mathrm{Ni}, \mathrm{Rh}, \mathrm{Pt}$ and $\mathrm{Co}$ has been studied in Fe-Pd alloys [11-19]. On studying the effect of Co and Ni addition on the Fe-Pd alloys, Tschiya et al. have shown recently that, independently of the Pd content, both the irreversible and irreversible MT temperatures decreased as the electron concentration, e/a, increased, so the compositional dependence of the MT temperatures of the studied Fe-Pd-X alloy seemed to be related to $e / a$ [13]. In this sense, one might think that lower e/a ratios could lead to higher transformation temperatures.

In this work the partial substitution of $\mathrm{Fe}$ by $\mathrm{Co}$ and $\mathrm{Mn}$ on the structural and magnetic properties of Fe-Pd alloys has been investigated. These substitutions lead to ternary alloys whose electron concentrations are higher and lower, respectively, than those on the binary alloy. It is found that the addition of $\mathrm{Mn}$ increases both the reversible and irreversible MT temperatures whereas the addition of Co causes the opposite effect, thus confirming the e/a ratio influence on the transformation temperatures. Nevertheless, the appearance of the BCT seems to be directly related to the tetragonality of the FCT phase. On the other hand, the amount of FCT martensite that irreversibly transforms into the undesirable BCT phase is considerably reduced by both the Co and Mn doping. With respect to the magnetic response, it is observed that the saturation magnetization and the magnetic anisotropy of the Fe-Pd alloys increase as a result of the Co addition whereas a slight decrease occurs due to the Mn addition.

\section{Experimental}

Polycrystalline ingots of $\mathrm{Fe}_{70} \mathrm{Pd}_{30}, \mathrm{Fe}_{67} \mathrm{Pd}_{30} \mathrm{Co}_{3}$ and $\mathrm{Fe}_{66.8} \mathrm{Pd}_{30.7} \mathrm{Mn}_{2.5}$ alloys were prepared from high purity elements by arc melting under protective Ar atmosphere. The ingots were homogenized in vacuum quartz ampoules at $1273 \mathrm{~K}$ during 24 hours and then subjected to a 30 minutes annealing treatment at $1173 \mathrm{~K}$ followed by quenching into ice water in a vertical furnace (the quenching process is necessary to get the metastable FCC austenitic phase). Once treated, the composition of the elaborated alloys was analyzed by EDS in a Jeol JSM-5610LV Scanning Electron Microscope (SEM). Small samples for calorimetric and magnetic measurements were obtained from discs previously cut from the centre of the ingots by slow speed diamond saw. In order to determine the transformation temperatures, DSC measurements were carried out at a heating/cooling rate of $10 \mathrm{~K} / \mathrm{min}$ in a TA Q100 calorimeter. The magnetic characterization (AC susceptibility and magnetization measurements) was performed by SQUID magnetometry (QD MPMS XL-7 SQUID). Neutron diffraction measurements were performed at the D1B installation at the Institute LaueLangevin. The diffraction measurements were carried out from room temperature down to $10 \mathrm{~K}$ and 
again up to room temperature at a cooling/heating rate of $1 \mathrm{~K} / \mathrm{min}$. The neutron wavelength was $1.28 \AA \AA$.

\section{Results and discussion}

Figure 1 shows the cooling curve of the DSC thermograms obtained on a cooling/heating ramp performed between RT and $125 \mathrm{~K}$ on the three elaborated alloys. In spite of the different shapes, a high temperature exothermic peak and a low temperature anomaly can be observed in each case. As indicated in the figure, the high and low DSC peaks are attributed to the FCC $\rightarrow \mathrm{FCT}$ and $\mathrm{FCT} \rightarrow \mathrm{BCT}$ transformations, respectively. This correspondence follows from the fact that once the alloys were cooled down to $125 \mathrm{~K}$ no peak was observed in the subsequent heating ramp, whereas a high temperature endothermic peak corresponding to the reverse FCT $\rightarrow$ FCC appears if the alloys are cooled to temperatures just above the low temperature anomaly. In the case of the binary and the Co-containing alloys the FCT $\rightarrow$ BCT transformation causes a series of DSC peaks (evidencing the burst-like character of such transformation) but only a small smooth peak is observed associated to the $\mathrm{FCT} \rightarrow \mathrm{BCT}$ transformation of the Mn-containing alloy.

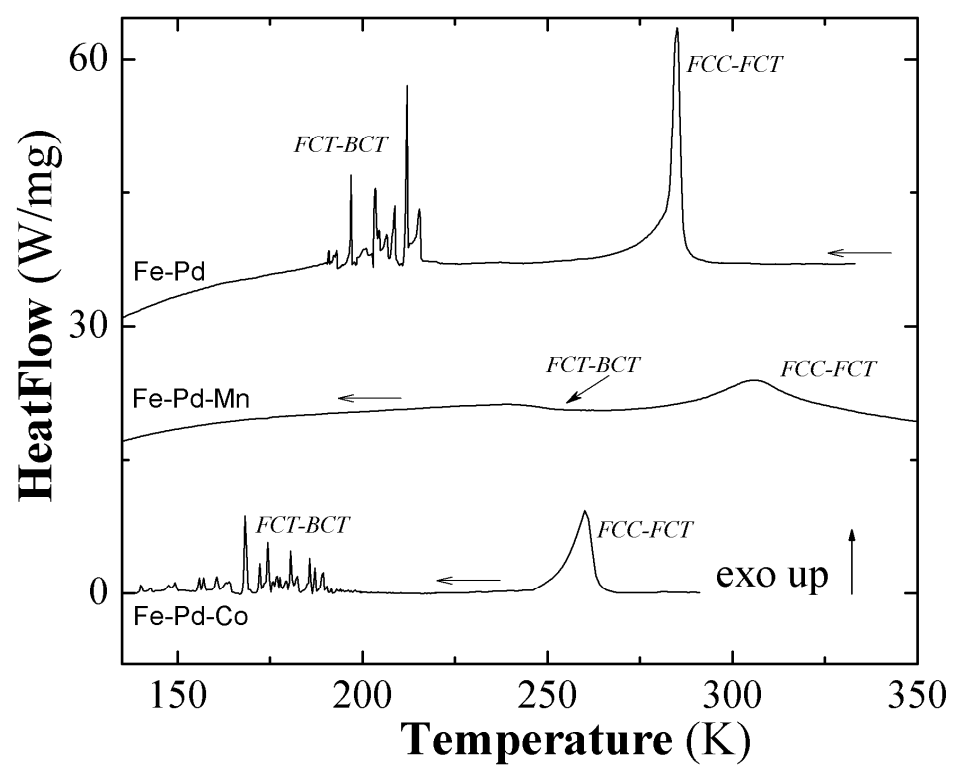

Fig. 1: Cooling curve of the DSC thermograms obtained on a cooling/heating ramp performed between RT and $125 \mathrm{~K}$ on the three elaborated alloys.

In order to study the compositional dependence of the transformation temperatures, the onset on cooling of the DSC high and low temperature peaks have been taken as the reversible and irreversible transformations temperatures ( $T_{\text {rev }}$ and $T_{\text {irrev }}$, respectively ). Figure 2 shows the transformation temperatures and the thermoelastic martensite temperature range $\left(T_{\text {rev }}-T_{\text {irrev }}\right)$ of the three alloys as a function of the electron concentration. Both transformation temperatures decrease as the $e / a$ ratio increases, independently of the Pd content on the alloy. Furthermore, the Fe-Pd-Mn alloy, which is the Pd-richest one, shows the highest transformation temperatures among them. Therefore, it seems that the compositional dependence of the transformation temperatures of these alloys is not related to the Pd amount but to the e/a concentration, just in accordance with the results in [13]. On the other hand, it is worth noting that the temperature range of stability of the FCT phase is slightly improved with both the Co and Mn addition. 


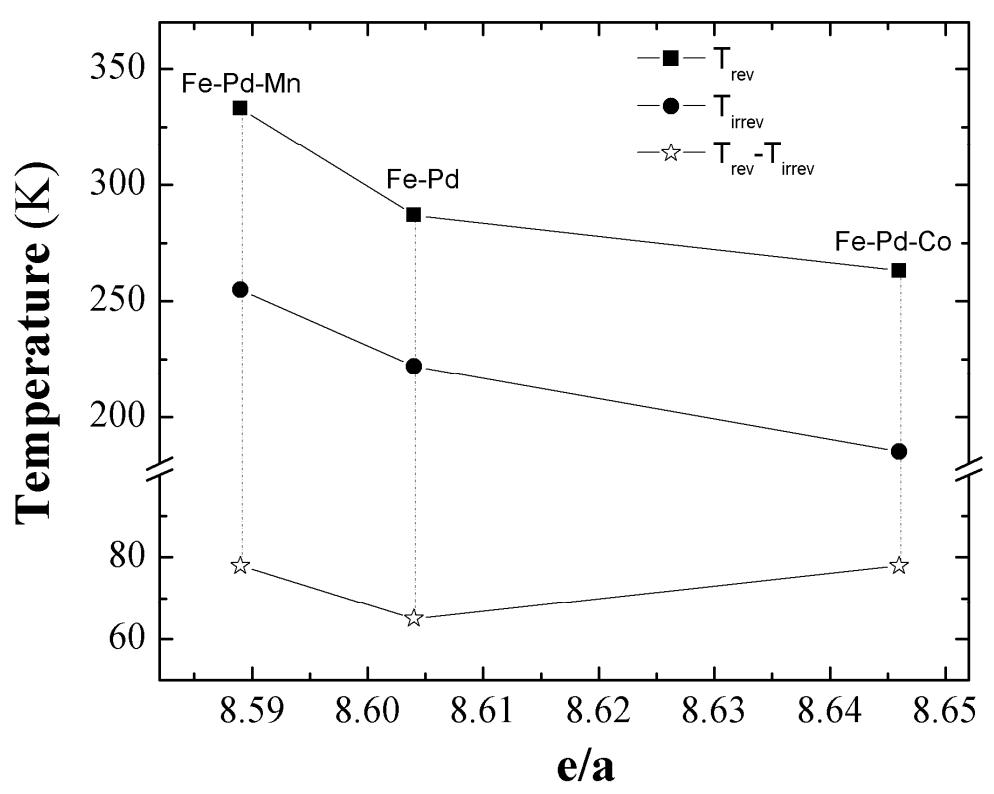

Fig. 2: Transformation temperatures and thermoelastic martensite temperature range $\left(T_{\text {rev }}-T_{\text {irrev }}\right)$ of the three alloys as a function of the electron concentration.

The lattice parameters of the alloy have been obtained from "in-situ" neutron diffraction measurements on a cooling ramp from $320 \mathrm{~K}$ down to $10 \mathrm{~K}$. The temperature dependence of both the lattice parameters and the tetragonality ( $c / a)$ of both the FCC and the FCT phases are shown in figure 3 as a function of the temperature. The three alloys show practically the same cubic lattice parameter ( $a \sim 5.725 \AA$ ) $)$, so no significant volume effect seems to arise from the Co and Mn addition. In the three cases, the cubic lattice parameter remains almost constant on cooling down to $\mathrm{T}_{\text {rev }}$. On the contrary, the lattice parameters of the FCT phases show a similar evolution with the temperature, mainly characterized by a gradual decrease of the tetragonality with the decreasing temperature. This behavior points out the weak first order character of the FCC $\rightarrow$ FCT transformations [20].

Interestingly, the lattice parameters of the binary and the ternary alloys are almost the same at the transformation temperatures. Focusing on the FCT-BCT transformation, it seems that the temperature of appearance of the BCT phase is determined by the lattice parameters of the FCT phase. In particular, the irreversible transformation occurs when the tetragonality of the FCT phases lies around $c / a_{\text {irrev }} \sim 0.925$. On the contrary, since the austenite cubic $a$ parameter remains constant in a quite large temperature range, $\mathrm{T}_{\text {rev }}$ must not be directly related to lattice constant. Therefore, $\mathrm{T}_{\text {rev }}$ and $\mathrm{T}_{\text {irrev }}$ seems to be controlled by the electron concentration and the lattice parameters, respectively. In this sense, the almost parallel evolution of $\mathrm{T}_{\text {rev }}$ and $\mathrm{T}_{\text {irrev }}$ with e/a shown in Fig.2 would indicate that the $c / a$ decreasing rate on cooling of the three alloys is quite similar. Nevertheless, this $c / a$ decreasing rate must be slightly lower on the binary alloy since this alloy shows the lowest $\mathrm{T}_{\text {rev }}-\mathrm{T}_{\text {irrev }}$ range among the three ones. 

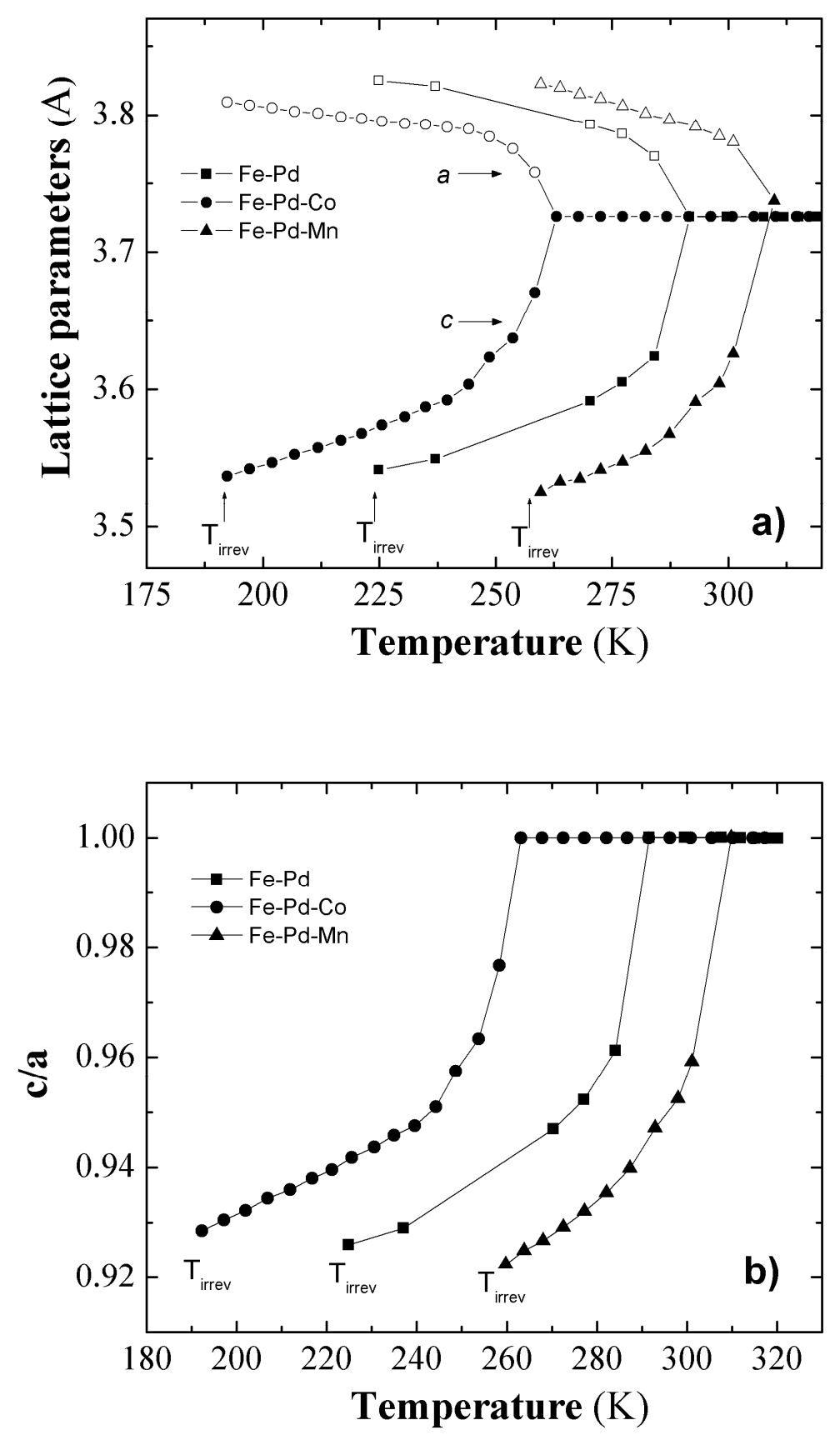

Fig. 3: Temperature dependence of both (a) the $a$ and $c$ lattice parameters and (b) the tetragonality of the FCT martensite on the three alloys.

The effect of Co and Mn doping on the volume fraction of FCT martensite transforming into the BCT phase has been also estimated from neutron diffraction measurements. Figure 4 shows the diffractograms obtained on the three alloys at $320 \mathrm{~K}$ before and after cooling them down to $10 \mathrm{~K}$. The diffractograms at $320 \mathrm{~K}$ before cooling corresponds exclusively to the FCC structure, but after cooling down to $10 \mathrm{~K}$ (below $\mathrm{T}_{\text {irrev }}$ ) the characteristic reflections corresponding to the $\mathrm{BCT}$ phase can be also observed. In this latter case the amount of austenite phase (and consequently the intensity of the FCC reflections) is lower due to the presence of the BCT phase. This loss of intensity on the FCC reflections must be then equal to the percentage of FCT phase transforming to the BCT structure [19]. By comparing the intensity of the $(220)_{\mathrm{FCC}}$ reflections before and after cooling, it is obtained that a $53 \%$, a $38 \%$ and a $32 \%$ of the mass of the Fe-Pd, Fe-Pd-Mn and Fe-Pd- 
Co alloys, respectively, transforms to the BCT martensite. Therefore, interestingly, the amount of undesirable BCT phase appearing in the binary alloy is reduced by doping in both cases.

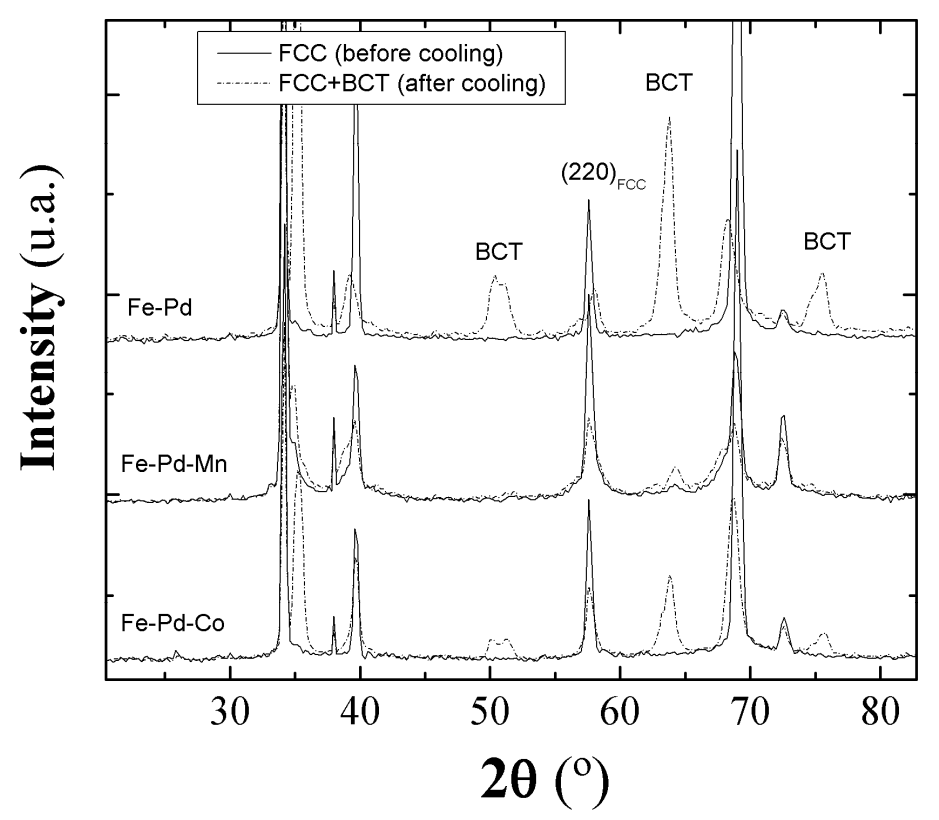

Fig. 4: Diffractograms at $320 \mathrm{~K}$ before and after cooling down to $10 \mathrm{~K}$ (solid and dashed lines, respectively).

In order to study the effect of Mn and Co doping on the magnetic response of the Fe-Pd alloy, low-field magnetic susceptibility and high-field magnetization measurements have been performed on the three alloys. The temperature dependence (cooling-heating-cooling) of the real part of the magnetic susceptibility $\left(\chi^{\prime}\right)$ is shown in figure 5 .

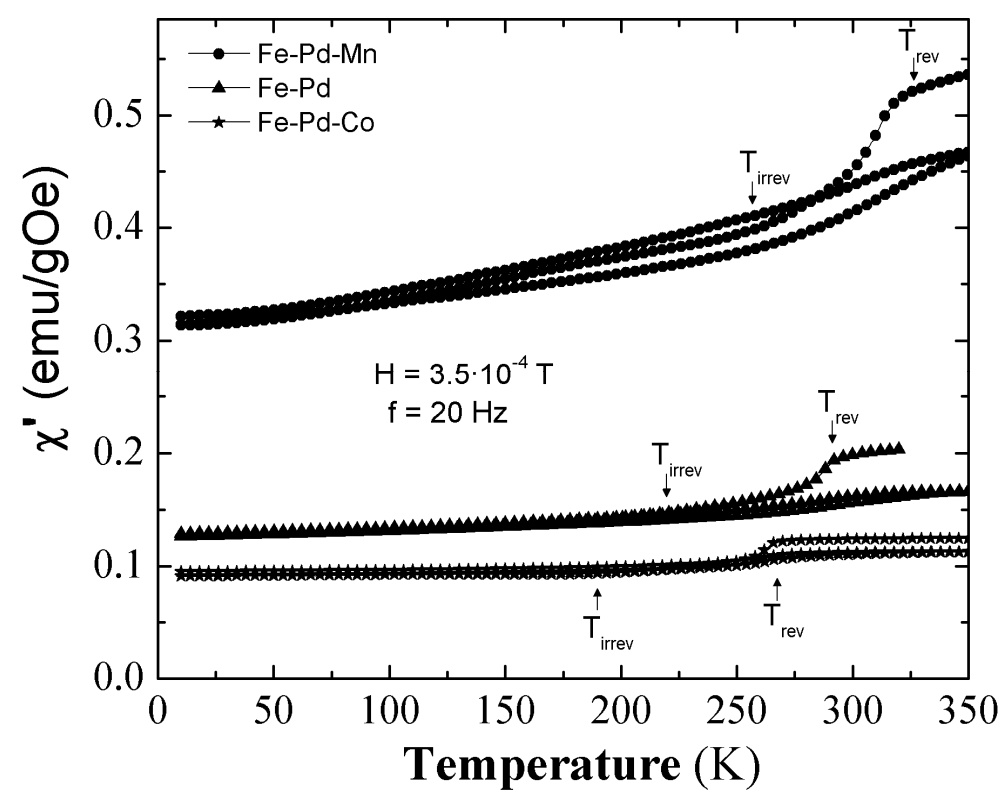

Fig. 5: Temperature dependence of the real part of the low-field magnetic susceptibility of the alloys. 
In the three cases a sudden change takes place at $\mathrm{T}_{\text {rev }}$ because of the $\mathrm{FCC} \leftrightarrow \mathrm{FCT}$ transformation (due to the higher magnetic anisotropy of the FCT phase) but in any case no anomaly associated to the FCT-BCT is observed at $\mathrm{T}_{\text {irrev }}$. This would mean that the magnetic anisotropy of the FCT and BCT phases of the studied alloys must be very similar. On the other hand, the fact that the initial value of $\chi^{\prime}$ at $350 \mathrm{~K}$ is not reached once the samples have been cooled below $\mathrm{T}_{\text {irrev }}$ must be ascribed to the presence of the BCT phase. It is also worth noting that the Mn addition makes the alloy be magnetically softer whereas the Co addition causes the opposite effect, which points out a respective decrease and increase of the magnetic anisotropy.

The magnetization under a 6T magnetic field has also been measured in the three alloys. In order to better compare the high-field magnetization of the different FCT phases (which highly determines the achievable MFIS), the measurements were performed on heating from above $T_{\text {irrev }}$. Figure 6 shows the magnetization of the FCT phase of the three alloys at 260K as a function of the e/a ratio. In spite of the Fe concentration is almost the same in both ternary alloys, it can be seen that the Co addition slightly increases the saturation magnetization $\left(\mathrm{M}_{\mathrm{S}}\right)$ of the FCT phase whereas a considerable decrease occurs as a consequence of the Mn doping.

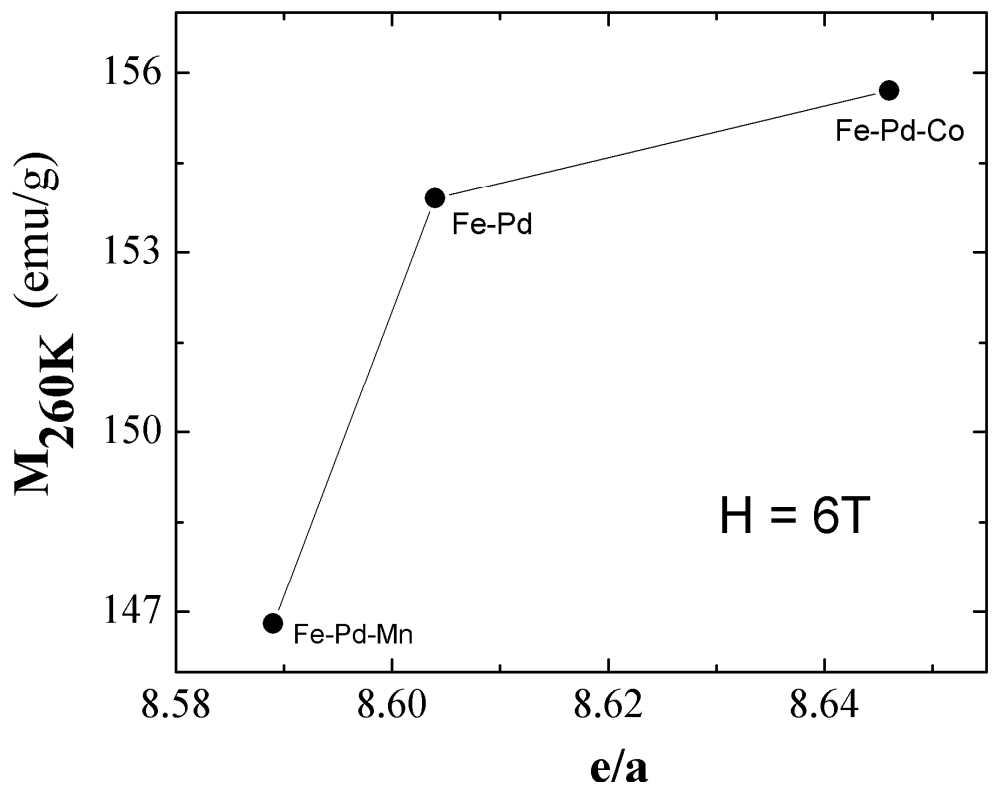

Fig. 6: Saturation magnetization of the FCT phase of the three alloys at $260 \mathrm{~K}$ as a function of the electron concentration.

From above magnetic measurements, it can be concluded that the substitution of Fe by $\mathrm{Co}$ is especially interesting for MFIS applications since both the magnetic anisotropy and the saturation magnetization of the Fe-Pd FCT martensite are considerably increased as a result of the cobalt addition.

\section{Conclusions}

The effect of partial substitution of Fe by Co and Mn (at.3\%, approximately) on the structural and magnetic properties of Fe-Pd FSMA has been investigated. It is found that the addition of $\mathrm{Mn}$ increases both the reversible and irreversible MT temperatures whereas the addition of Co causes the opposite effect. Independently of the Pd concentration, the transformation temperatures increase as the electron concentration decrease, so the compositional dependence of such temperatures seems to be mainly related to the e/a ratio. Besides, in both cases, the temperature range of stability of the FCT martensite (and therefore the temperature range for MFIS) is also increased by doping. On the other hand, it is found that the temperature of appearance of the BCT phase in these Fe-Pd- $(\mathrm{Mn} / \mathrm{Co})$ alloys is directly related to the tetragonality of the FCT phase. However, no significant volume 
effects seem to arise from the Co and Mn addition. From neutron diffraction measurements it is also found that the amount of undesirable BCT phase appearing in the binary alloy is reduced with both $\mathrm{Mn}$ and Co doping. With respect to the magnetic properties, the substitution of Fe by Co seems to be especially interesting for MFIS applications since both the magnetic anisotropy and the saturation magnetization of the Fe-Pd FCT martensite are considerably increased.

\section{Acknowledgements}

This work has been carried out with the financial support of the Spanish "Ministerio de Ciencia y Tecnología" (Project number MAT2006-12838) and the Government of Navarra (Project entitled "Efecto magnetocalórico en aleaciones con memoria de forma ferromagnéticas"). The Institute Laue Langeving, D1B installations (Grenoble, France), is acknowledged for the allocated neutron beamtime (Exp. 5-24-262). The authors are grateful to C. Gómez-Polo for helpful discussions.

\section{References}

[1] R.C. O’Handley, S.J. Murray, M. Marioni, H. Nembach, S.M. Allen: J. Appl. Phys. Vol. 87 (2000), p.4712.

[2] T. Krenke, E. Duman, M. Acet, E.F. Wassermann, X. Moya, Ll. Mañosa, A. Planes: Nature Mater. Vol. 4 (2005), p. 450.

[3] Z.H. Liu, H. Liu, X.X. Zhang, X. K. Zhang, J.Q. Xiao, Z.Y. Zhu, X.F. Day, G. D. Liu, J.L. Chen, G.H. Gu: Appl. Phys. Lett. Vol. 86 (2005), p. 182507.

[4] A. Sozinov, A.A. Likhachev, N. Lanzka, K. Ullakko: Appl. Phys. Lett. Vol. 80 (2002), p. 746

[5] H. Kato, Y. Liang, M. Taya: Scr. Mater. Vol. 46 (2002), p. 71.

[6] T. Fukuda, T. Sakamoto, T. Kakeshita, T. Takeuchi, K. Kishio: Mater. Trans. Vol. 4 (2004), p.188.

[7] R.D. James, M. Wuttig: Philos. Mag. A Vol. 77 (1998), p.1273.

[8] M. Sugiyama, R. Oshima, F.E. Fujita: Trans. JIM Vol. 19 (1984), p.585.

[9] J. Cui, T.W. Shield, R.D. James: Acta Mater. Vol. 52 (2004), p. 35.

[10] V.A. Chernenko, E. Cesari, V.V. Kokorin, I.N. Vitenko: Scr. Metall. Mater. Vol. 33 (1995), p. 1239.

[11] R.A. Stern, S.D. Wiiloughby, A. Ramirez, J.M. Maclaren, J. Cui, Q. Pan, R.D. James: J. Appl. Phys. Vol. 91 (2002), p.7818.

[12] K. Tanaka, K. Hiraga, R. Oshima: Mater. Trans. JIM Vol. 33 (1992), p.215.

[13] K. Tsuchiya, T. Nojiri, H. Ohtsuka, M. Umemoto: Mater. Trans. JIM Vol. 44 (2003), p.2499.

[14] G.S. Yang, R. Jonasson, S.N. Baek, K. Murata, S. Inoue, K. Koterazawa, S.J. Jeong, K. Mizuuchi, K. Inoue: Mater. Dev. Smart Syst. Vol. 785 (2004), p.475.

[15] T. Wada, T. Tagawa, M. Taya: Scr. Mater. Vol. 48 (2003), p. 207.

[16] D. Vokoun, Y.W. Wang, T. Goryczka, C.T. Hu: Smart. Mat. Struct. Vol. 14 (2005), p. 261.

[17] Y.C. Lin, H.T. Lee, S.U. Jen, Y.T. Chen: J Appl. Phys. Vol. 101 (2007), p.09N514.

[18] S.U. Jen, Y.T. Chen, T.L. Tsai, Y.C. Lin: J. Appl. Phys. Vol. 103 (2008), p.07B902.

[19] V. Sánchez-Alarcos, V. Recarte, J.I. Pérez-Landazábal, C. Gómez-Polo, V.A. Chernenko, M.A. González: Eur. Phys. J. ST Vol. 158 (2008), p.107.

[20] R. Oshima: Scr. Metall. Vol. 15 (1981), p.829. 